\title{
Topology of $\mathrm{SmB}_{6}$ determined by dynamical mean field theory
}

\author{
P. Thunström $\oplus^{1,2}$ and K. Held ${ }^{1}$ \\ ${ }^{1}$ Institute of Solid State Physics, TU Wien, 1040 Vienna, Austria \\ ${ }^{2}$ Department of Physics and Astronomy, Materials Theory, Uppsala University, 75120 Uppsala, Sweden
}

(Received 8 July 2019; revised 11 July 2021; accepted 15 July 2021; published 17 August 2021)

\begin{abstract}
Whether $\mathrm{SmB}_{6}$ is a topological insulator remains hotly debated. Our density functional theory plus dynamical mean field theory calculations are in excellent agreement with a large range of experiments, from the $4 f^{5.5}$ intermediate valency to $\mathrm{x}$-ray and photoemission spectra. We show that $\mathrm{SmB}_{6}$ is a strongly correlated topological insulator, albeit not a Kondo insulator, using a symmetry analysis of the "pole extended" Hamiltonian, which fully captures the effect of the self-energy arising from the strong electron-electron correlation within the $\mathrm{Sm} 4 f$ orbitals. The topological surface states are analyzed, addressing conflicting interpretations of photoemission data.
\end{abstract}

DOI: 10.1103/PhysRevB.104.075131

\section{INTRODUCTION}

The discovery of topology in the electronic band structure has added a whole new dimension to solid state physics $[1,2]$. One of the most striking manifestations of a nontrivial topology is the emergence of robust metallic surface states in topological insulators. Such topological behavior has been established for the semiconductors mercury telluride [3] and bismuth selenide $[4,5]$, but the situation remains unclear for most other materials. For semiconductors, theory [6] is often ahead of experiment. That is, there are quite reliable predictions based on density functional theory (DFT) $[7,8]$ but no clear-cut experimental validation. Much more difficult, particularly for theory, are strongly correlated insulators for which the one-electron band picture breaks down.

The archetype of such strongly correlated insulators is $\mathrm{SmB}_{6}$, which was proposed by Dzero et al. [9] to be a topological Kondo insulator based on a (Kondo renormalized) noninteracting band structure and the topological $Z_{2}$ invariant as per Ref. [10]. However, the strong correlations and intermediate valency of $\mathrm{SmB}_{6}$ give rise to an intricate multiplet structure [11] with no clear adiabatic connection to a noninteracting system. Predictions based on a Kondo renormalization or the "topological Hamiltonian" [12] of the system, constructed from the self-energy at zero frequency, may hence break down [13]. In this article we will therefore, instead of the topological Hamiltonian, focus on the less studied "pole extended" (PE) Hamiltonian [14], which fully captures the physical spectral function, and hence is a rigorous starting point for the topological classification [15].

On the experimental side, there is clear evidence of robust metallic surface states, such as a surface-dependent plateau in the low-temperature resistance $[16,17]$ and an-

Published by the American Physical Society under the terms of the Creative Commons Attribution 4.0 International license. Further distribution of this work must maintain attribution to the author(s) and the published article's title, journal citation, and DOI. Funded by Bibsam. gular resolved photoemission spectroscopy (ARPES) data [18-22], including the spin texture of $\mathrm{SmB}_{6}$ [23]. However, the states' topological origin has been questioned [21,24]. Indeed, whether the low-energy electronic properties even originate from the surface or the bulk remains hotly debated. De Haas-van Alphen oscillations have been interpreted as stemming from both the bulk [25] and the surface [26,27]. The same holds for the main ARPES features: Ref. [28] vs Refs. $[11,27]$. The low-temperature linear specific heat has been shown to be predominantly a bulk effect [29], and the same holds for the optical conductivity within the band gap [30]. On the other hand, it is was argued [31] that these effects are not intrinsic but stem from ${ }^{154} \mathrm{Gd}$ impurities which elude a mass purification against ${ }^{154} \mathrm{Sm}$.

This controversy clearly calls for a better theoretical understanding. Because of the strong electronic correlations and well-localized $4 f$ orbitals, DFT + dynamical mean field theory (DMFT) [32-34] is the method of choice. There have been earlier DFT+DMFT [35-37], DFT+impurity [38,39], and DFT+Gutzwiller [40] calculations which however did not capture the bulk band gap, the flatness of the $f$ bands, and the intermediate valence of $\mathrm{SmB}_{6}$ all at the same time [11], due to various additional approximations. Indeed, the combination of these properties poses a hard theoretical challenge that requires an accurate many-body treatment of the $\mathrm{Sm} 4 f$ orbitals.

In this article, we present charge self-consistent DFT+DMFT calculations for $\mathrm{SmB}_{6}$. Our results provide an all-encompassing picture of the bulk and surface properties of $\mathrm{SmB}_{6}$, in excellent agreement with many experimental observations. Analyzing the symmetry properties of the corresponding PE Hamiltonian $[14,15]$ we show that $\mathrm{SmB}_{6}$ is a strongly correlated topological insulator.

\section{DFT+DMFT METHOD}

All calculations have been performed using the relativistic spin polarized toolkit (RSPt) [41-44], which is based on linearized muffin tin orbitals. This method allows the correlated $\mathrm{Sm} 4 f$ orbitals to be readily identified and projected upon in the DMFT calculation [42]. The full Coulomb interaction, spin-orbit interaction, and local Hamiltonian for 


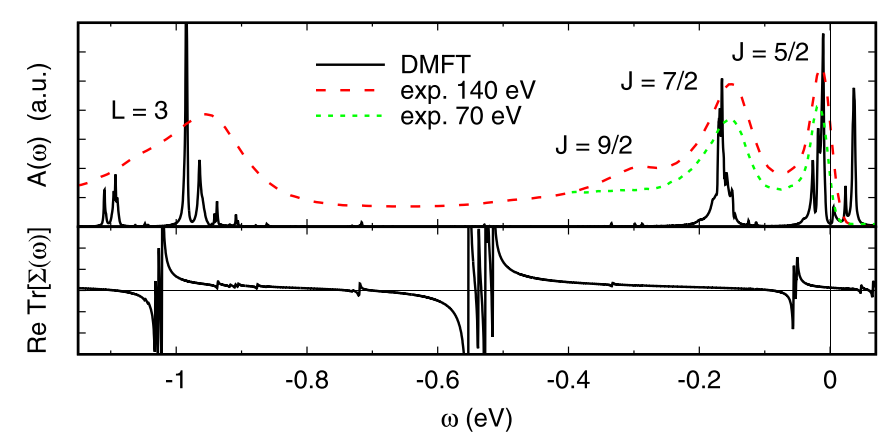

FIG. 1. Upper panel: Bulk k-integrated DFT+DMFT spectrum of $\mathrm{SmB}_{6}$ at $100 \mathrm{~K}$ compared to experimental photoemission data [11]. Sm $4 f^{6} \rightarrow 4 f^{5}$ transitions with the $J$ and $L$ quantum numbers of the final $\left(4 f^{5}\right)$ state are indicated. Lower panel: The trace of the local self-energy (real part) shows distinct clusters of poles (with the shape $[\omega-E]^{-1}$ ), but not within the bulk band gap around $\omega=E_{F}=0$.

the $\mathrm{Sm} 4 f$ orbitals are included in the DMFT impurity problem, which is solved using the exact diagonalization (ED) approach $[43,45,46]$. The bulk calculations were performed at $T=25 \mathrm{~K}, 50 \mathrm{~K}, 75 \mathrm{~K}$, and $100 \mathrm{~K}$ and iterated until charge self-consistency [47]. The charge self-consistent DFT+DMFT slab calculations were due to the large computational cost at low temperatures only performed at $100 \mathrm{~K}$. For additional details, such as the final bath state parameters and the double counting procedure [48,49], see Appendix A.

\section{RESULTS}

\section{A. Bulk}

The hybridization between the strongly localized Sm $4 f$ orbitals and the surrounding orbitals is too weak to form a coherently screened (Kondo) ground state at temperatures down to $25 \mathrm{~K}$. Instead we find an intermediate valence state with a thermal mixture of both $\operatorname{Sm~} 4 f^{6}$ and $4 f^{5}$ configurations, and an average $4 f$ occupation of $n_{f} \approx 5.5$, in good agreement with experiment [50]. The $4 f^{6}$ contribution is predominately of $\Gamma_{1}$ character and the $4 f^{5}$ of $\Gamma_{8}$ in agreement with nonresonant inelastic $x$-ray (NIXS) data [51]. The almost temperature-independent $4 f$ occupation is the result of a feedback between the thermal occupation and the chemical potential of the system. The chemical potential gradually reduces the energy difference between the $\Gamma_{1}$ many-body state and the four $\Gamma_{8}$ many-body states in the thermal ground state as the temperature is lowered, which keeps the $4 f$ occupation almost constant. This makes the results, up to a small shift in the Fermi energy, almost temperature independent in the range from $25 \mathrm{~K}$ to $100 \mathrm{~K}$. A detailed characterization of the thermal ground state is given in Appendix B 1.

The k-integrated spectral function (DOS) reflects the intermediate valence and displays distinct $4 f^{6} \rightarrow 4 f^{5}$ and $4 f^{5} \rightarrow 4 f^{4}$ multiplet transitions, as shown in Fig. 1 and Appendix B 2, respectively. The first peak below the Fermi energy $\left(E_{F}\right)$ at $-11 \mathrm{meV}$ is to a ${ }^{6} H_{5 / 2} \Gamma_{8}$ final state, as shown in Appendix B 1. Upon closer inspection, we notice that the low-energy $\Gamma_{8}$ peak has a small shoulder around $-19 \mathrm{meV}$, and that there is a small peak at $-28 \mathrm{meV}\left(\Gamma_{7}\right)$; both are too small to be well resolved in the experiment.

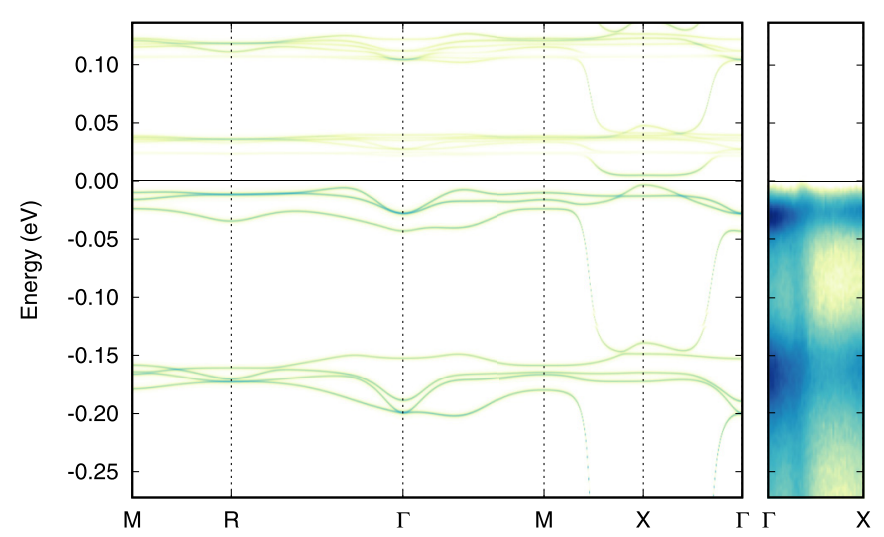

FIG. 2. Left: Bulk DFT+DMFT band structure of $\mathrm{SmB}_{6}$ along the indicated high-symmetry path through the Brillouin zone. Right: Experimental ARPES data [11] along $\Gamma-X$. The hybridization between the dispersive $\mathrm{Sm} 5 d+\mathrm{B} 2 p$ band and the flat $\mathrm{Sm} 4 f$ bands gives rise to a $9 \mathrm{meV}$ bulk band gap around the $X$ point.

The next peak around $-170 \mathrm{meV}$ corresponds to $J=7 / 2$ final states $\left({ }^{6} H_{7 / 2}\right)$. The experimental on-resonance spectrum $(\hbar v=140 \mathrm{eV})$ displays an additional broad $J=9 / 2$ derived shoulder around $-300 \mathrm{meV}$. This feature is hardly discernible in theory or at off-resonance conditions $(\hbar v=70 \mathrm{eV})$ since the direct transitions from the $J=04 f^{6}$ configuration to the $J=9 / 24 f^{5}$ configurations, i.e., without an intermediate core excitation, are largely forbidden.

The sharp multiplet peaks in the spectrum are generated from a many-body self-energy with a large number of distinct poles, as seen in Fig. 1 (lower panel), in contrast to the linear self-energy of the Kondo renormalization scenario. However, there is no pole within the narrow bulk band gap, as one would have for a Mott insulator. The bulk band gap is instead a hybridization gap, caused by the small hybridization between the Sm $4 f$ orbitals and the dispersive B $2 p$ and Sm $5 d$ orbitals. We will come back to the poles of the self-energy when discussing the topological properties.

Let us now turn to the momentum-resolved spectral function in Fig. 2 (left). The Sm multiplet transitions discussed above result in almost flat $f$ bands each carrying a small fraction of the total weight. The very weak dispersion splits the $\Gamma_{8}$-derived bands slightly, which gives rise to the shoulder at $-19 \mathrm{meV}$ seen in the DOS. The Sm $5 d$ orbitals on the other hand hybridize strongly with the B $2 p$ orbitals and form a parabolic band centered at the $X$ point. When this dispersive band approaches the flat $f$ bands close to the $E_{F}$ the hybridization results in a small band gap of about $9 \mathrm{meV}$ (16 meV peak-to-peak), close to the experimental value of $\sim 10-20 \mathrm{meV}[11,52]$. The spectral function agrees well with the bulk-sensitive ARPES experiments of Denlinger et al. [11] reproduced in Fig. 2 (right).

Altogether, we find that the local DFT+DMFT self-energy gives an accurate description of the experimentally established bulk properties of $\mathrm{SmB}_{6}$. Hence we can now turn to its topological properties with confidence.

\section{B. Proof of nontrivial topology}

The topological $Z_{2}$ invariant of Kane and $\mathrm{Fu}$ [10] can be determined, for an inversion-symmetric 
noninteracting system, from the parity $\mathcal{P}_{\mathbf{k}_{i} ; m}$ of the (pairs of Kramers degenerate) bands $m$ below $E_{F}$ at the time-reversal invariant moment (TRIM) momenta $\mathbf{k}_{i}$. If $\prod_{i m} \mathcal{P}_{\mathbf{k}_{i} ; m}=-1$, the system has a nontrivial topology. However, this procedure cannot be directly applied to an interacting system, as the one-particle self-energy can split, smear out, and reduce the weights of the bands, and even make them fade away and reappear at shifted energies. Several suggestions of how to generalize the $Z_{2}$ invariant to the interacting case have been made [12,15], but as common in the fast-moving field of topological materials, the theory still needs to be developed in full.

The self-energy consists in general of a set of poles located on the real frequency axis [53], clearly shown in Fig. 1 (bottom). The local self-energy can hence be written as

$$
\Sigma_{m n}(\omega)=\Sigma_{m n}(\infty)+\sum_{l} V_{m l}^{\dagger}\left[\omega-E_{l}\right]^{-1} V_{l n}
$$

Here, $E_{l}$ and $V_{m l}^{\dagger} V_{l n}$ are the pole position and weight, respectively, and $m, n$ are the local spin-orbital indices. This pole structure of the self-energy is from a mathematical point of view identical to that of an additional fictitious hybridization function where the physical orbitals $m$ hybridize via $V_{l m}$ with a large number of local auxiliary orbitals with energy $E_{l}$, one auxiliary orbital per pole. That is, we can make an exact mapping, akin to a purification of a mixed state, of the interacting bulk system with the self-energy $\Sigma_{m n}$ to a noninteracting "pole extended" (PE) Hamiltonian with additional orbitals $l$ [14]. As in the case of a purification, we recover the physical band structure by simply projecting the PE band structure onto the physical orbitals. As this PE Hamiltonian is effectively noninteracting, the topological invariant $Z_{2}$ of Ref. [10] can be straightforwardly applied [15]. If this $Z_{2}$ is nontrivial, the PE Hamiltonian has topological surface states, which manifest themselves in the physical system through the projection onto the physical orbitals.

In the following, we show that $\mathrm{SmB}_{6}$ is a topological insulator by proving that

(1) its PE Hamiltonian is topologically nontrivial, and

(2) the resulting topological surface states have a finite physical weight. The arguments are grounded in a symmetrybased analysis of the PE Hamiltonian which makes the topological classification very robust against potential imprecisions in our DFT+DMFT calculation.

As for (1), the construction of the PE Hamiltonian requires the full pole structure of the self-energy, which can be difficult to obtain. Nevertheless, we can still assess its topological properties from some general considerations:

(i) As shown in Fig. 1, the self-energy has no pole at the Fermi energy.

(ii) The DMFT self-energy is k-independent in its local basis, which means that the PE bands must have a finite physical weight to be dispersive.

From (i) and (ii) it follows that since there is a finite band gap in the known physical spectral function, the PE Hamiltonian is insulating as well. Note that a purely auxiliary band, which is not visible in the physical spectral function, cannot cross the band gap as this requires a finite dispersion. A third physically motivated condition, given the large bandwidth of the $\mathrm{Sm} 5 d$ and B $2 p$ derived dispersive band, is

(iii) Only the correlated Sm $4 f$ states carry a DMFT selfenergy; the Sm $5 d$ and B $2 p$ orbitals have $\Sigma=0$.

This point is of practical importance, as it will allow us to evaluate the $Z_{2}$ invariant of the PE Hamiltonian by the standard approach of counting eigenstates (bands) with a given parity at the TRIM points. The reason is that the auxiliary orbitals given by a local self-energy inherit the irreducible representation of the local correlated orbitals they derive from [54]. In our case, the auxiliary orbitals have the same odd parity [55] as the $\mathrm{Sm} 4 f$ orbitals. The orbitals of opposite (even) parity will therefore form noninteracting bands at the TRIM points. It is therefore enough to count these (Kramers pairs of) noninteracting bands with even parity at the TRIM points to evaluate the $Z_{2}$ invariant. These are $6,6,5$, and 4 at $\Gamma, R, X$, and $M$, respectively [56]. The physical reason for the odd number at the $X$ point is that the dispersive band of Sm $5 d$ and B $2 p$ character, which is the only band with even parity in the vicinity of the Fermi energy, is below $E_{F}$ at $X$ while above $E_{F}$ at the other TRIM points. This implies that $\prod_{i m} \mathcal{P}_{\mathbf{k}_{i} ; m}=-1$; i.e., the PE Hamiltonian is topologically nontrivial.

This nontrivial topology is very robust against potential imprecisions in our DFT+DMFT calculation: They would need to shift an even-parity orbital to the other side of $E_{F}$ at an odd number of TRIM points, which would require the band with $\mathrm{Sm} 5 d$ and B $2 p$ character to shift by several eV, while maintaining the mixed valency. Furthermore, this shift must originate from the DFT potential and hence the electron density as $\Sigma=0$ for this noninteracting even-parity orbital.

As for (2): The nontrivial topology of the PE Hamiltonian in the bulk implies robust metallic surface states in the extended Hilbert space spanned by both the physical orbitals and the auxiliary orbitals. The remaining question is whether the topological surface states may have a completely auxiliary character, or whether they must carry a finite physical weight as they cross the bulk band gap. The DMFT self-energy, which provides a good description of $\mathrm{SmB}_{6}$, varies from layer-tolayer perpendicular to the surface, but it still fulfills (ii). The $\mathrm{PE}$ bands must therefore have a finite physical weight to be dispersive, which is necessary to cross the bulk band gap. Hence a nontrivial topology in the bulk implies robust metallic surface states with finite weight if the self-energy is local.

\section{Surface states}

To address the conflicting interpretations of the experimental photoemission data $[11,20,21]$, we performed additional DFT+DMFT slab calculations. The [001] $\mathrm{SmB}_{6}$ supercell, shown to the right in Fig. 3, was used with two different terminations to represent the different surface patches of cleaved samples $[11,21]$. The first considered supercell has a terminating $\mathrm{B}_{6}$ layer (depicted without bonds) and the outermost $\mathrm{Sm}$ atom is $\mathrm{Sm}^{3+}$, while the second supercell lacks the $\mathrm{B}_{6}$ layer and is instead terminated by $\mathrm{Sm}^{2+}$ surface atoms. The $\mathrm{B}_{6}$ termination has trivial surface bands associated with a $\mathrm{B}_{6}$ dangling bond [24] that disappears when the surface reconstructs $[11,21,24]$. To effectively mimic this partial surface reconstruction in our computationally expensive DMFT 

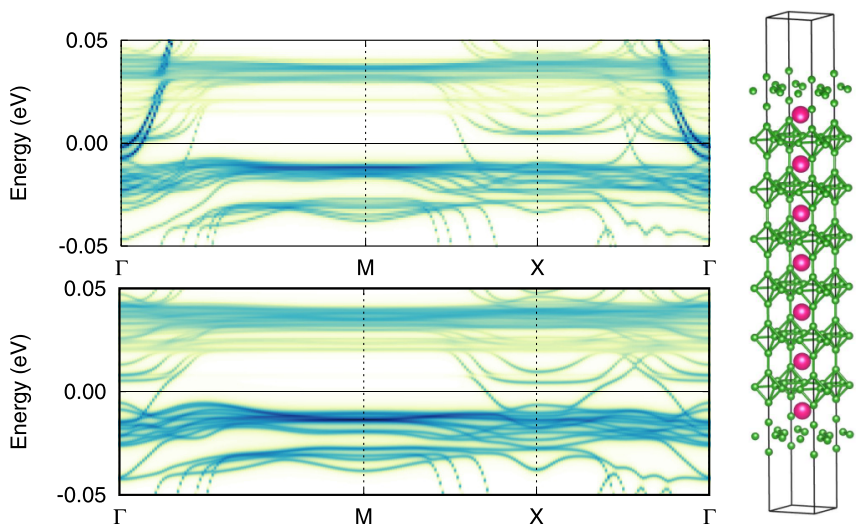

FIG. 3. DFT+DMFT spectrum resolved for $k_{x}$ and $k_{y}$ of a periodic $\mathrm{SmB}_{6}$ supercell with (top left) and without (bottom left) $\mathrm{B}_{6}$ surface termination. The former is depicted on the right. Both terminations show topological surface states that cross the bulk band gap. The $\mathrm{B}_{6}$ termination has additional trivial parabolic surface bands centered at the $\Gamma$ point associated with the $\mathrm{B}_{6}$ dangling bond.

calculations, we simply apply an additional $0.5 \mathrm{eV}$ potential to the $2 p$ orbitals of the outermost $\mathrm{B}$ atom in the final step. This potential shifts the trivial surface band, but does not directly affect the topological surface states which live, as we will see, in the subsurface layer.

The resulting DFT+DMFT spectra are presented in Fig. 3 (left). We recover the flat $\mathrm{Sm} 4 f$ bands of the bulk at $-14 \mathrm{meV}$ and $+40 \mathrm{meV}$ as well as the $m_{J}= \pm 1 / 2, J=5 / 2$ band directly above $E_{F}$ at the $X$ point. On top of these bulk states surface bands emerge within the bulk band gap. These metallic surface bands are mainly associated with the subsurface Sm layer, as the $\mathrm{Sm} 4 f$ states of the outermost Sm layer are shifted away from $E_{F}$ due to their pure $\mathrm{Sm}^{2+}$ or $\mathrm{Sm}^{3+}$ characters. In Appendix $\mathrm{C}$ we further confirm the topological protection of the surface bands by applying artificial potentials to the subsurface atoms. The topological surface states simply shift deeper into the material when an additional (time-reversal symmetric) perturbing potential is applied to the (sub)surface layer. When the topological protection is lifted by an out-of-plane magnetic field, which breaks the time-reversal symmetry, the surface bands can hybridize with each other and form a band gap, as shown in Appendix C.

Figure 4 shows cuts of the spectral function at $E_{F}$ and $-5 \mathrm{meV}$ below. The surface states form large and intermediate sized pockets around both the $X$ and $\Gamma$ points for the $\mathrm{B}_{6}$ and $\mathrm{Sm}$ termination, respectively. The $X$ pockets show a strong spin polarization in agreement with spin-resolved ARPES experiments [20]. Reference [21] reports both the large $X$ and $\Gamma$ pockets for the $\mathrm{B}_{6}$-terminated surface, although they interpret the large $\Gamma$ pocket as an umklapp state. The trivial "Rashba split" surface states, which are reported close to the $\Gamma$ point directly after cleaving [21], seem instead to correspond to the shifted $\mathrm{B}_{6}$ derived trivial surface bands. Reference [11] obtains intermediate sized $X$ pockets and picks up holelike $\mathrm{Sm} 4 f$ dispersion around the $H$ points for an aged mainly B-terminated surface, as reproduced in Fig. 4, but as in Refs. $[18,20]$ the $\Gamma$ pocket is seemingly missing. However, on closer inspection of the data, in particular in the

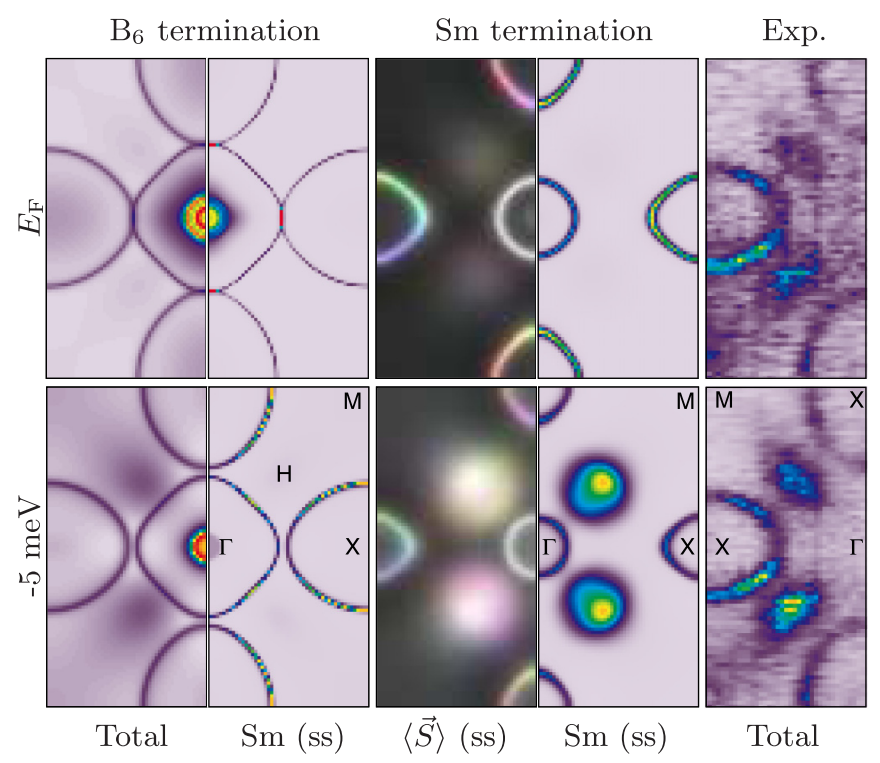

FIG. 4. Surface states resolved for $k_{x}$ and $k_{y}$ at $E_{F}$ (top) and $-5 \mathrm{meV}$ below $E_{F}$ (bottom). Left: $\mathrm{B}_{6}$ termination. Middle: $\mathrm{Sm}$ termination. Right: Experiment [11]. The $\mathrm{B}_{6}$ termination shows the total and $\mathrm{Sm} 4 f$ subsurface (ss) contribution. The Sm termination shows the Sm $4 f$ (ss) contribution and its spin projection $\langle\vec{S}\rangle$ mapped on the color wheel. The maximum total intensity for the $\mathrm{B}_{6}$ termination (leftmost panel) is approximately 7 times larger than its Sm (ss) projection.

second Brillouin zone, there is clear evidence of a matching surface-derived $\Gamma$ pocket, which again has been interpreted as an umklapp state $[18,20]$. Hence, to finally settle the apparent discrepancy between theory and experiment, we support a critical experimental reexamination of this umklapp assignment [22]. For example, our data for the Sm termination suggest that the $\Gamma$ pocket has a much weaker spin polarization than the $X$ pocket, and that a surface reconstruction could make it extend deeper into the bulk compared to the $X$ pocket as shown in Appendix C.

\section{CONCLUSION}

The appropriateness of DFT+DMFT for describing the strong correlations in $\mathrm{SmB}_{6}$ and the excellent description of various experimental properties give us confidence that we have achieved an accurate theoretical description of $\mathrm{SmB}_{6}$. We determined its topology in a rigorous way from the symmetry properties of the PE Hamiltonian, formed by mapping the pole structure of the self-energy onto a set of auxiliary orbitals $[14,15]$. We prove that for a local DMFT self-energy with a given parity it is enough to count the bands of the opposite-parity channel at the TRIM points, under the condition that the self-energy does not have a pole at $E_{F}$. This band analysis shows that $\mathrm{SmB}_{6}$ constitutes a robust topological insulator.

In general, if the positions of the bands in one parity channel are well described by DFT, then the addition of a local self-energy in the other parity channel will not change the topology of the system as long as it remains insulating. If the local self-energy has poles within the bulk band gap 
topological surface bands will still appear in the spectral function, but they may gradually flatten out and lose spectral weight as they approach the poles. Nevertheless, between the poles they will always carry a finite weight. Our data suggest however that the "missing" $\Gamma$ pocket in $\mathrm{SmB}_{6}$ is not due to poles in the band gap but that it simply has been interpreted as an umklapp state $[18,20,21]$.

\section{ACKNOWLEDGMENTS}

We are highly indebted to J. D. Denlinger and J. W. Allen for making available their ARPES data. We would also like to thank Annica Black-Schaffer and Dushko Kuzmanovski for useful discussions. This work has been financially supported in part by European Research Council under the European Union's Seventh Framework Program (FP/2007-2013) through ERC Grant Agreement No. 306447. The computations were performed in part on resources provided by SNIC, under project SNIC 2018/3-489.

\section{APPENDIX A: COMPUTATIONAL DETAILS}

All the $\mathrm{SmB}_{6}$ DFT+DMFT calculations were performed using the experimental cubic crystal structure (space group: 221, prototype: $\mathrm{CaB}_{6}$ ) with the lattice parameter $a=4.13$ $\AA$. The DFT exchange-correlation functional was set to the local density approximation (LDA) [8], and the Brillouin zone was sampled through a conventional Monkhorst-Pack mesh of $16 \times 16 \times 16 k$ points in the bulk and $16 \times 16 \times 16 k$ points in the slab calculations. The local Coulomb interaction was parametrized in terms of the Slater parameters $F^{0}, F^{2}, F^{4}$, and $F^{6}$. The Hubbard $U$ parameter $F^{0}$ is heavily screened by the valence electrons and was set to $8.0 \mathrm{eV}$. The less screened Slater parameters $F^{2}, F^{4}$, and $F^{6}$ were instead calculated through Slater integrals at the beginning of each DFT iteration, and then scaled by $0.95,0.97$, and 1.00 , respectively [44]. The final self-consistent values are given in Table I.

TABLE I. Screened Sm $4 f$ Slater integrals $\left(F^{0}-F^{6}\right)$ and the double counting potential $\left(\mu_{\mathrm{DC}}\right)$ of the Sm atoms in the bulk and the Sm-terminated $(\mathrm{Sm})$ and $\mathrm{B}_{6}$-terminated $\left(\mathrm{B}_{6}\right)$ supercells. The latter is depicted in Fig. 3, and the former in Fig. 10. The labels start from the middle Sm atom (Sm1) and go toward the surface (Sm2-Sm4). The $\mathrm{Sm}$ atoms have an intermediate valence except the outermost $\mathrm{Sm}$ atom which is $\mathrm{Sm}^{2+}\left(\mathrm{Sm}^{3+}\right)$ in the slab with $\mathrm{Sm}\left(\mathrm{B}_{6}\right)$ termination. The fully localized limit double counting potential $\left(\mu_{\mathrm{DC}}^{\mathrm{FLL}}\right)$ is given as a reference. All values are given in $\mathrm{eV}$.

\begin{tabular}{lccccccc}
\hline \hline Label & Termination & $F^{0}$ & $F^{2}$ & $F^{4}$ & $F^{6}$ & $\mu_{\mathrm{DC}}$ & $\mu_{\mathrm{DC}}^{\mathrm{FLL}}$ \\
\hline $\mathrm{Sm}$ & Bulk & 8.0 & 11.43 & 7.49 & 5.54 & 35.32 & 37.75 \\
$\mathrm{Sm} 1$ & $\mathrm{Sm}$ & 8.0 & 11.39 & 7.47 & 5.52 & 35.55 & 37.82 \\
$\mathrm{Sm} 2$ & $\mathrm{Sm}$ & 8.0 & 11.40 & 7.47 & 5.52 & 35.55 & 37.91 \\
$\mathrm{Sm} 3$ & $\mathrm{Sm}$ & 8.0 & 11.39 & 7.47 & 5.52 & 35.70 & 37.85 \\
$\mathrm{Sm} 4$ & $\mathrm{Sm}$ & 8.0 & 10.83 & 7.07 & 5.22 & 39.62 & 41.75 \\
$\mathrm{Sm} 1$ & $\mathrm{~B}_{6}$ & 8.0 & 11.43 & 7.50 & 5.54 & 35.25 & 37.72 \\
$\mathrm{Sm} 2$ & $\mathrm{~B}_{6}$ & 8.0 & 11.43 & 7.50 & 5.54 & 35.26 & 37.67 \\
$\mathrm{Sm} 3$ & $\mathrm{~B}_{6}$ & 8.0 & 11.42 & 7.49 & 5.54 & 35.29 & 37.75 \\
$\mathrm{Sm} 4$ & $\mathrm{~B}_{6}$ & 8.0 & 11.86 & 7.80 & 5.77 & 32.33 & 34.37 \\
\hline \hline
\end{tabular}

The fully charge self-consistent DFT+DMFT calculations were performed using the full-potential linear muffin tin orbital (LMTO) code RSPt [41] and the DMFT implementation presented in Refs. [42,43,47]. The lattice Hamiltonian as well as the DMFT lattice Green's function are calculated in the full LMTO basis. The projection operators upon the localized Sm $4 f$ orbitals are obtained from a k-dependent Löwdin orthogonalization of the LMTO Sm $4 f$ orbitals, as detailed in the Supplemental Material of Ref. [43]. The self-energy is calculated explicitly for each Sm atom in the supercells used in the slab calculations.

The Sm $5 d$ and B $2 p$ orbitals form a parabolic band centered at the $X$ point. The band starts at $-2 \mathrm{eV}$ below the Fermi energy and spans more than $15 \mathrm{eV}$ through a folding of the Brillouin zone at $+7 \mathrm{eV}$. These orbitals are also very extended compared to the contracted Sm $4 f$ orbitals, which implies a stronger screening of the Coulomb interaction. Hence, their effective Coulomb interaction is much smaller than their kinetic energy. In addition, the intermediate valence of the $\mathrm{Sm} 4 f$ make the occupation of the dispersive band noninteger, which further suppress the effects of electronic correlations. We have therefore only added a self-energy to the $\mathrm{Sm} 4 f$ orbitals in the DFT+DMFT calculations, as the Sm $5 d$ and B $2 p$ orbitals are already well described by the DFT Hamiltonian.

\section{Exact diagonalization}

The $\mathrm{Sm} 4 f$ orbitals are strongly contracted compared to the Sm $5 d$ and $6 s$ orbitals, which makes the Sm $4 f$ orbitals hybridize very weakly with the orbitals on neighboring atoms. The hybridization is completely captured by the local hybridization function $\Delta(\omega)$ [32-34] that describes how the electrons propagate in the material once they leave the $\mathrm{Sm} 4 f$ orbitals of a given atom. To put the weak Sm $4 f$ hybridization in perspective: the $\mathrm{Ni} 3 d$ hybridization function in $\mathrm{NiO}$ is about 20 times larger than the $\mathrm{Sm} 4 f$ hybridization function in $\mathrm{SmB}_{6}$ for comparable computational setups. The electronic structure of $\mathrm{SmB}_{6}$ is therefore already well described on the eV energy scale by completely neglecting the hybridization [38]. However, this approach is not accurate enough to describe the important meV energy scale close to the Fermi energy. It is particularly important that the hybridization function at the Fermi energy $\Delta(0)$ is accurately captured, as well as the $\mathrm{Sm} 4 f$ occupation, to ensure that the Fermi energy remains in the hybridization band gap during the DFT+DMFT selfconsistency cycle. The exact diagonalization (ED) impurity solver $[43,45,46]$ takes most of the hybridization between the correlated $\mathrm{Sm} 4 f$ orbitals and the rest of the material into account by including a limited number of effective bath orbitals in the impurity problem. However, the total number of bath orbitals that can be included in the impurity problem is severely limited by the exponential growth of the many-body Hilbert space. In order to still get an accurate representation of $\Delta(0)$ and the $\mathrm{Sm} 4 f$ occupation we need to go beyond the standard hybridization fitting scheme as well as modifying the double counting correction. The details thereof are described in the next two subsections. 


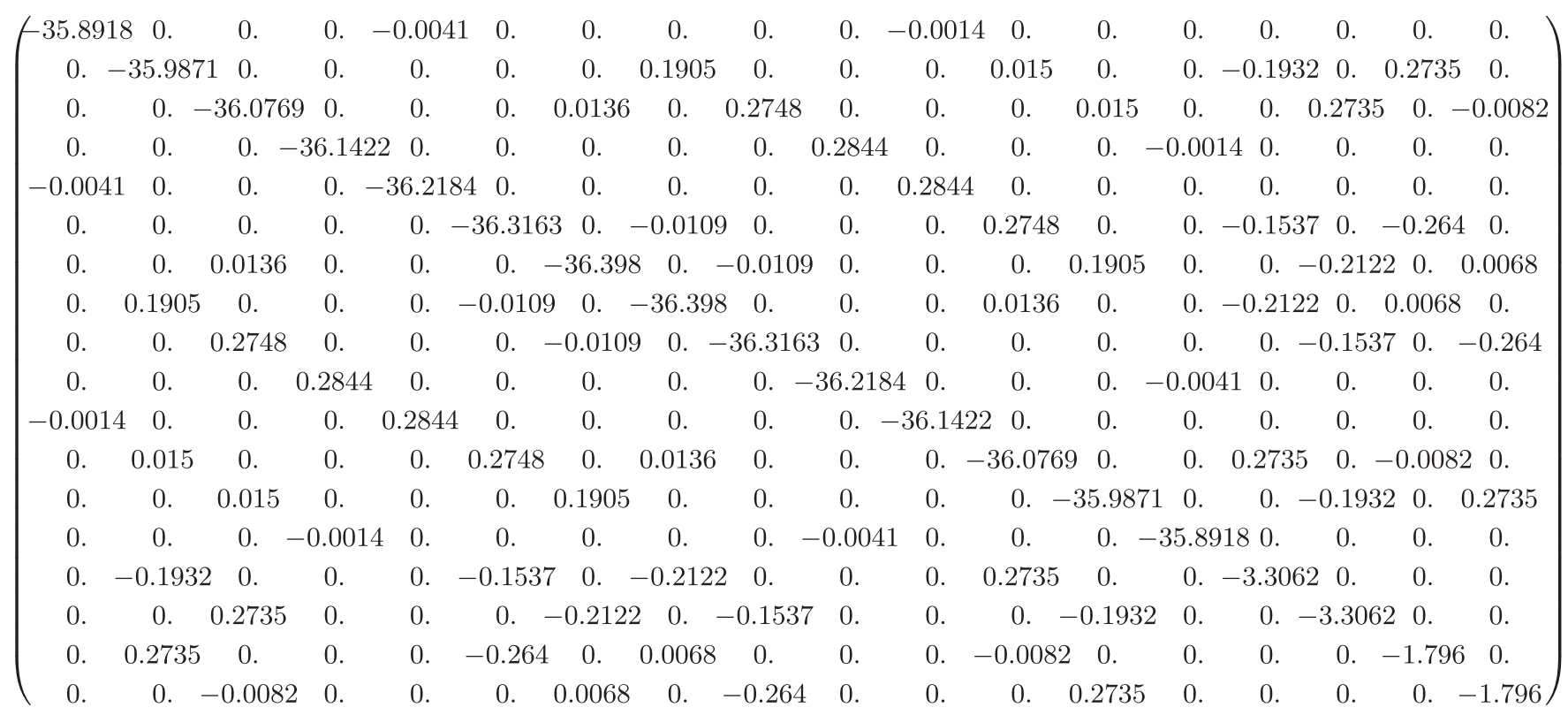

FIG. 5. One-particle term $H_{0}^{E D}$, including the double counting correction and the down-folded high-energy bath states, of the self-consistent $\mathrm{Sm} 4 f$ ED Hamiltonian of $\mathrm{SmB}_{6}$ (bulk). The $14 \mathrm{Sm} 4 f$ spin orbitals are located in the upper left corner and ordered according to $\left(l_{z}, s_{z}\right)=$ $(-3,-1 / 2),(-2,-1 / 2), \ldots,(3,-1 / 2),(-3,1 / 2), \ldots,(3,1 / 2)$. The remaining 4 spin orbitals in the lower right corner are the bath states. The energy unit is eV.

\section{Bath discretization}

In DMFT, the lattice self-energy is replaced with periodically repeated copies of the local self-energy $\Sigma(\omega)$. The bulk lattice Green's function $G_{k}(\omega)$ is hence given by

$$
G_{k}(\omega)=\left[\omega-H^{k}-\Sigma(\omega)\right]^{-1},
$$

where the $k$ point $k$ belongs to the first Brillouin zone. In the self-consistent DMFT scheme the lattice Green's function in Eq. (A1) is projected onto the local $\operatorname{Sm} 4 f$ orbitals $(\mathcal{F})$ and integrated over the Brillouin zone to yield the local Green's function $G_{F F}(\omega)$. The hybridization function is then extracted from the matrix inverse of $G_{F F}(\omega)$,

$$
\Delta(\omega)=\omega \mathbf{1}_{F F}-H_{F F}-\Sigma_{F F}(\omega)-\left[G_{F F}(\omega)\right]^{-1},
$$

where $H_{F F}$ is the matrix representation of the locally projected Hamiltonian which includes the spin-orbit coupling, the double counting, and all crystal field terms, and the matrix $\Sigma_{F F}(\omega)$ is the self-energy projected onto the local $\mathrm{Sm} 4 f$ orbitals. The ED method proceeds by fitting a few bath state parameters in the matrices $H_{F B}$ and $H_{B B}$ to $\Delta(\omega)$ via the model function

$$
\Delta^{E D}(\omega)=H_{F B}\left[\omega \mathbf{1}_{B B}-H_{B B}\right]^{-1} H_{B F},
$$

where $H_{B F}=H_{F B}^{\dagger}$. We fit the hybridization function on the Matsubara axis with 6 bath states per Sm orbital using a conjugate gradient scheme which also takes the off-diagonal terms in $\Delta(\omega)$ into account. The high-energy bath states $\left(\mathcal{B}^{\prime}\right)$, i.e., the eigenstates of $H_{B B}$ with energies $E_{b^{\prime}}$ relatively far from the Fermi energy $\left(\left|E_{b^{\prime}}\right| \gg w_{b^{\prime}} \equiv \sqrt{H_{b^{\prime} F} H_{F b^{\prime}}}\right)$, give only a perturbative contribution to the low-energy physics due to the large energy cost of exciting these bath states. Their effect can be estimated by introducing the scaling $H_{B^{\prime} B^{\prime}} \rightarrow \lambda H_{B^{\prime} B^{\prime}}$ and $H_{F B^{\prime}} \rightarrow \sqrt{\lambda} H_{F B^{\prime}}$ in $\Delta^{E D}(\omega)$, which keeps $\Delta^{E D}(0)$ invariant, and let the scaling parameter $\lambda \rightarrow \infty$. The scaling shows that the high-energy bath states can be replaced to zeroth order by a static contribution

$$
\begin{aligned}
\Delta^{E D}(\omega) \approx & H_{F B^{\prime \prime}}\left[\omega \mathbf{1}_{B^{\prime \prime} B^{\prime \prime}}-H_{B^{\prime \prime} B^{\prime \prime}}\right]^{-1} H_{B^{\prime \prime} F}^{\dagger} \\
& +H_{F B^{\prime}}\left[-H_{B^{\prime} B^{\prime}}\right]^{-1} H_{B^{\prime} F}^{\dagger},
\end{aligned}
$$

where $B^{\prime \prime}$ is the low-energy complement to $B^{\prime}$, i.e., $B=B^{\prime \prime}+$ $B^{\prime}$. In our calculations we put the high-energy cutoff at $\left|E_{b^{\prime}}\right|>$ $10 w_{b^{\prime}}$. The remaining low-energy bath states were ordered according to their weight $w_{b}$, and included in $\mathcal{B}^{\prime \prime}$ to the extent allowed by the computational resources, with a minimum of 4 bath states in total. The converged one-particle term of the ED Hamiltonian $H_{0}^{E D}=H_{F F}+H_{F B}+H_{B F}+H_{B B}$ for the bulk calculation is presented in Fig. 5.

\section{Double counting correction}

In the DFT+DMFT scheme, the explicit addition of a local Coulomb interaction term to the DFT Hamiltonian introduces a double counting (DC) of the electron-electron interaction. The unknown form of the screening processes in the exchange correlation functional prevents the implementation of an exact double counting correction. In particular, the screening of the local interaction between the $\mathrm{Sm} 4 f$ electrons, implicitly described within the LDA, may be different from the effective (static) screening implied by the renormalized Slater parameters. Due to this ambiguity several different double counting corrections schemes have been suggested over the years, such as the fully localized limit (FLL) [48] and around mean field (AMF) [49]. The FLL correction removes the spherically averaged Hartree-Fock contribution of an effective atomic-like system with integer orbital occupations. The AMF correction considers instead an effective itinerant system with 
TABLE II. Lowest energy many-body eigenstates of the impurity Hamiltonian obtained at $100 \mathrm{~K}$. The symmetry (Sym), Sm $4 f$ occupation $\left(N_{f}\right)$, energy $(E)$, and thermal weight (GS) are tabulated for each eigenstate, as well as their contribution to the $\operatorname{Sm~} 4 f$ total $(J)$, angular $(L)$, and spin $(S)$ moment.

\begin{tabular}{|c|c|c|c|c|c|c|c|c|c|}
\hline Sym & $N_{f}$ & $E(\mathrm{meV})$ & GS (\%) & $J$ & $L$ & $S$ & $J_{z}$ & $L_{z}$ & $S_{z}$ \\
\hline$\Gamma_{1}^{+}$ & 6.002 & 0 & 46 & 0.03 & 2.95 & 2.94 & 0.00 & 0.00 & 0.00 \\
\hline$\Gamma_{8}^{-}$ & 5.030 & 11 & 12 & 2.52 & 4.93 & 2.47 & 1.80 & 3.16 & -1.36 \\
\hline$\Gamma_{8}^{-}$ & 5.030 & 11 & 12 & 2.52 & 4.93 & 2.47 & -0.47 & -0.75 & 0.28 \\
\hline$\Gamma_{8}^{-}$ & 5.030 & 11 & 12 & 2.52 & 4.93 & 2.47 & -1.80 & -3.16 & 1.36 \\
\hline$\Gamma_{7}^{-}$ & 5.029 & 24 & 3 & 2.53 & 4.93 & 2.47 & 0.77 & 1.07 & -0.30 \\
\hline$\Gamma$ & 6.002 & 47 & 0 & 1.01 & 2.96 & 2.95 & -1.00 & -0.50 & -0.50 \\
\hline$\Gamma$ & 6.002 & 47 & 0 & 1.01 & 2.96 & 2.95 & 0.00 & 0.00 & -0.00 \\
\hline$\Gamma$ & 6.002 & 47 & 0 & 1.01 & 2.96 & 2.95 & 1.00 & 0.50 & 0.50 \\
\hline
\end{tabular}

uniform (noninteger) orbital occupations. However, the thermal ground state of an intermediate valence compound such as $\mathrm{SmB}_{6}$ falls outside these two scenarios as it contains several thermally occupied almost atomic-like many-body eigenstates having different numbers of electrons, as shown in Table II in Appendix B 1 .

The occupation of an intermediate valence ground state responds to the DC potential in Fermi-Dirac function-like steps. However, the underlying assumptions of both FLL and AMF make their DC correction linear in the occupation. The two different behaviors always lead to a feedback loop away from intermediate valence toward integer valence. A second, less severe issue is that the finite discretization of the bath states in ED causes a small mismatch between the impurity Green's function and the local Green's function $G_{F F}(\omega)$ projected from the lattice. To minimize these two problems we automatically adjusted the double counting potential at each DMFT iteration to obtain the same number of Sm $4 f$ electrons on the impurity as in the lattice. This procedure makes the occupation of the $\mathrm{Sm} 4 f$ orbitals in the bulk stabilize to $n_{f}=5.49$ at a temperature of $100 \mathrm{~K}$, and to $n_{f}=5.50$ at $75 \mathrm{~K}, 50 \mathrm{~K}$, and $25 \mathrm{~K}$. The experimentally observed $\mathrm{Sm}$ $4 f$ occupation stays constant at $n_{f}=5.50 \pm 0.01$ between $15 \mathrm{~K}$ to $50 \mathrm{~K}$, after which it increases linearly to $n_{f}=5.43$ at $130 \mathrm{~K}$ [50]. The increase is linked to a transition in the phonon energy from a maximum at $50 \mathrm{~K}$ to a minimum at $130 \mathrm{~K}$ [50], which is an effect outside the scope of our calculations. The computationally expensive slab calculations were performed at $100 \mathrm{~K}$ to speed up their convergence and reduce their memory footprint. In these calculations we chose to enforce the bulk Sm $4 f$ occupation of 5.49 for all the $\mathrm{Sm}$ atoms except at the surface, to make the center $\mathrm{Sm}$ atom as bulklike as possible and minimize the hybridization between the surface states located at the top and bottom of the slab.

\section{APPENDIX B: MANY-BODY ELECTRONIC STRUCTURE}

\section{Thermal ground state}

The lowest energy many-body eigenstates of the selfconsistent impurity problem Hamiltonian $H^{E D}$ are given in Table II. The states have almost atomic-like Sm $4 f$ occupations $\left(N_{f}\right)$ and total angular momenta $(J)$, but the crystal fields and the hybridization with the bath mix the different $J_{z}$ configurations. The thermal ground state is an incoherent mixture of the eigenstates according to their Boltzmann weights (GS) $e^{-\beta E} / \operatorname{Tr}\left[e^{-\beta H}\right]$ at $100 \mathrm{~K}$. At this temperature the largest contributions are given by the four degenerate $\Gamma_{8}^{-}$states $(48 \%)$ with $J \approx 5 / 2$ and $N_{f} \approx 5$, and the $\Gamma_{1}^{+}$state $(46 \%)$ with $J \approx 0$ and $N_{f} \approx 6$, in agreement with nonresonant inelastic $\mathrm{x}$-ray scattering data [51]. The remaining $6 \%$ belongs to a $\Gamma_{7}^{-}$doublet.

In the slab calculations the surface introduces a tetragonal symmetry breaking that lifts the degeneracy of the four $\Gamma_{8}^{-}$ many-body states into two pairs of degenerate states. Depending on the surface termination the $\Gamma_{8}^{-}$states of the subsurface and sub-subsurface $\mathrm{Sm}$ atoms split by about $2-7 \mathrm{meV}$, which contributes to the multitude of bands seen in Fig. 3. The nontrivial topology of the electronic structure of the insulating bulk, i.e., the middle of the slab, is not affected by the surface potential. However, the dispersion of the topological surface states does depend on the details of the surface potential, as explicitly demonstrated in Appendix $\mathrm{C}$ with the help of additional perturbing potentials.

\section{Bulk photoemission spectra}

In Fig. 1 we have already shown the spectral function in an intermediate energy range from $-1.2 \mathrm{eV}$ to $0.1 \mathrm{eV}$, which resolves the $J$ and $j_{z}$ multiplets for $L=5$ and $L=3$. In Fig. 6 and Fig. 7 we show the same k-integrated spectral function but in a smaller and larger energy window, respectively. Clearly the peaks obtained with DFT+DMFT agree well with the experimentally measured (on-resonance) photoemission data [11], which is not the case for the $\operatorname{Sm} 4 f$-projected density of states of plain LDA, as shown in Fig. 6. The relative weights of the $4 f^{6} \rightarrow 4 f^{5}$ transitions are also accurately captured. The relative weights of the different $4 f^{5} \rightarrow 4 f^{4}$ transitions between $-5 \mathrm{eV}$ and $-12 \mathrm{eV}$ in Fig. 7 are in reasonable agreement with the experimental data, even though we do not include the resonance effect which strongly enhances their total weight. This gives us further assurance that our DFT+DMFT calculation faithfully describes bulk $\mathrm{SmB}_{6}$.

The shape of the Sm $4 f$-projected spectral functions at $25 \mathrm{~K}, 50 \mathrm{~K}, 75 \mathrm{~K}$, and $100 \mathrm{~K}$ is very similar, as shown in Fig. 6, up to a shift in the chemical potential. The shift is linked to a 


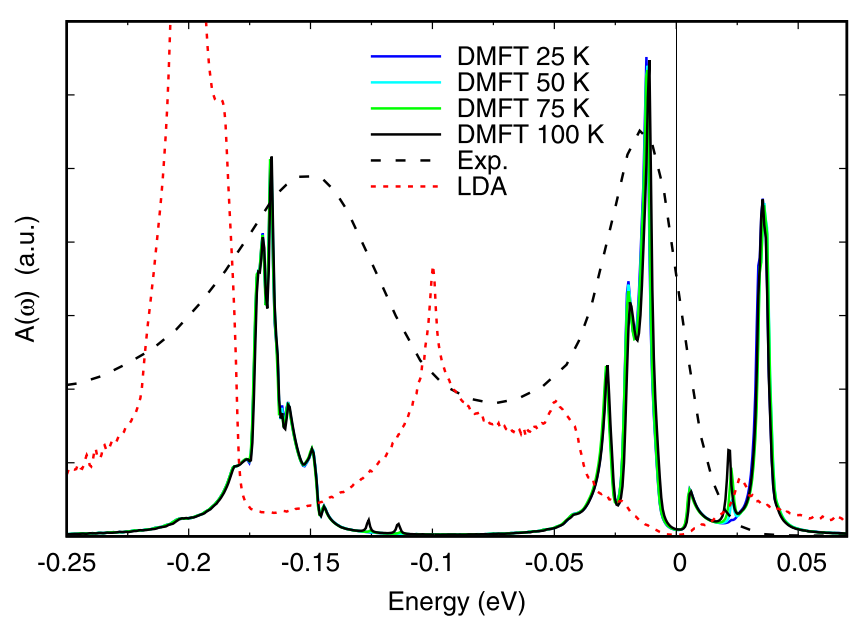

FIG. 6. Sm $4 f$-projected DFT+DMFT spectral density of $\mathrm{SmB}_{6}$ at the temperatures $25 \mathrm{~K}, 50 \mathrm{~K}, 75 \mathrm{~K}$, and $100 \mathrm{~K}$, compared to the Sm $4 f$-projected LDA density of states and experimental photoemission data [11]. To more clearly visualize the almost temperature-independent shape of the $\mathrm{Sm} 4 f$ spectral density, the DMFT spectra at $25 \mathrm{~K}, 50 \mathrm{~K}$, and $75 \mathrm{~K}$ have been aligned to the spectrum at $100 \mathrm{~K}$. Here, the alignment simply compensates for the drift of the chemical potential toward the top of the valence bands as the temperature is decreased.

reduction of the energy difference between the $\Gamma_{1}$ many-body state and the four $\Gamma_{8}$ many-body states in the thermal ground state. The only other difference is a suppression of a small peak at about $23 \mathrm{meV}$ in the unoccupied part of the spectrum. The small peak corresponds to transition from a thermally excited $\Gamma_{7}$ state $\left(n_{f}=5, E=24 \mathrm{meV}\right)$ to an $n_{f}=6$ state at $E=47 \mathrm{meV}$, both listed in Table II. Since the calculated electronic structure of the bulk is so similar within this temperature range, and the small differences are well understood, we are confident that the results from our slab calculations at $100 \mathrm{~K}$ also give an accurate description of the electronic structure at lower temperatures.

The main temperature-dependent effect observed in the experimental photoemission spectrum [35] between 25-100 K is a smearing of the bands, and as a result a reduced effective hybridization between the dispersive $\mathrm{Sm} 5 d+\mathrm{B} 2 p$ band and the flat $\mathrm{Sm} 4 f$ bands. Most of the smearing starts to set in at around $40-50 \mathrm{~K}$, which coincides with a transition in the phonon energy from a maximum at $50 \mathrm{~K}$ to a minimum at $130 \mathrm{~K}$ according to Ref. [50], which is an effect that is outside the scope of our electronic structure calculations. However, since the experimental bulk band gap is open at $25 \mathrm{~K}$, and the character of the bands does not change with thermal smearing, it is not essential to take this effect into account in our analysis of the topological character of the low-temperature electronic structure.

\section{APPENDIX C: ROBUSTNESS OF THE TOPOLOGICAL SURFACE STATES}

\section{Adding a surface potential}

To numerically confirm the topological protection of the surface bands we applied time-reversal symmetric potentials to the (sub)surface layer of the Sm-terminated supercell

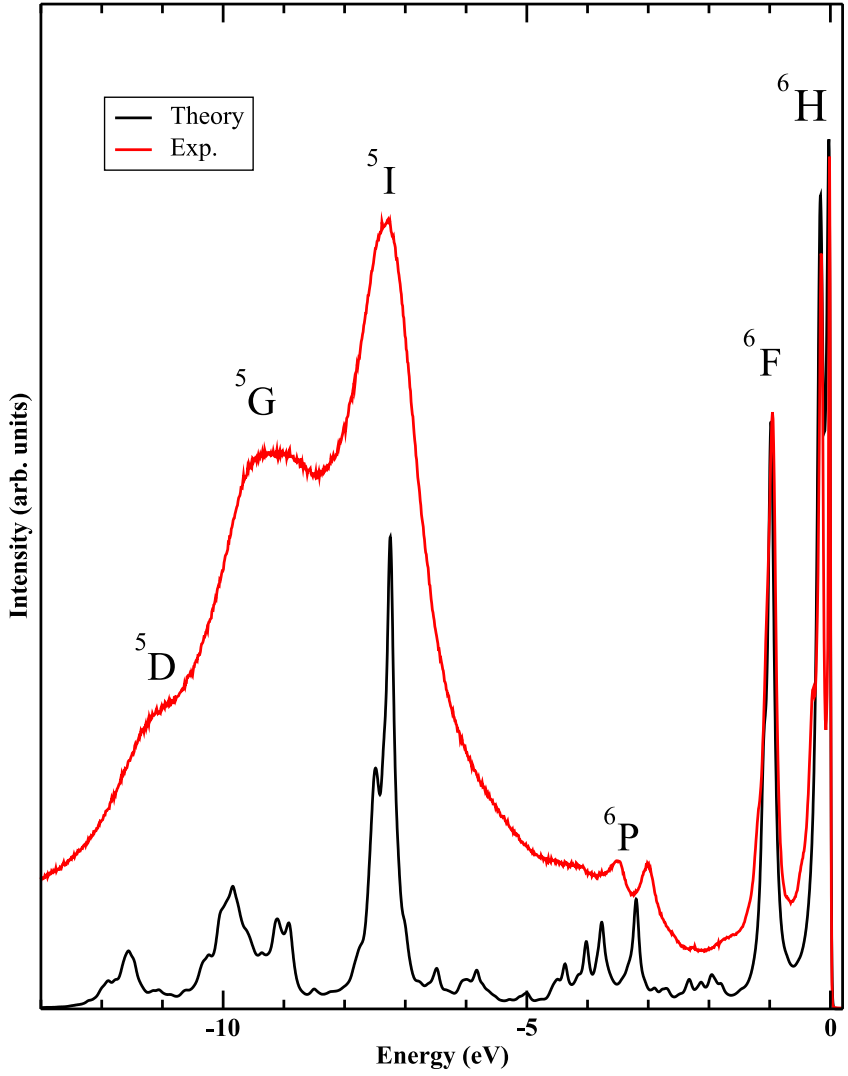

FIG. 7. Sm $4 f$-projected DFT+DMFT spectral density of $\mathrm{SmB}_{6}$ compared to experimental on-resonance $(\hbar v=140 \mathrm{eV})$ photoemission data [11]. The approximate $J$ and $L$ quantum numbers of the final states are indicated.

displayed to the right in Fig. 10. The topological surface states should survive under these perturbing potentials, which can occur for example due to surface reconstructions. Our results are shown in Figs. 8 and 9, where we applied the potential to the $j_{z}= \pm 5 / 2$ and $j_{z}= \pm 1 / 2$ spin orbitals of the $J=5 / 2$ Sm $4 f$ manifold, respectively. These spin orbitals were chosen as the surface states around the $\Gamma$ point have primarily $j_{z}= \pm 5 / 2$ character, while the surface states around the $X$ point have mainly $j_{z}= \pm 1 / 2$ character. Technically, we simply added the potential term to the self-energy of these states after DMFT convergence (a self-consistency including this potential is beyond our illustrative purposes). Both figures show the Sm $4 f$ projection on the subsurface (left panel) and sub-subsurface states (right panel). Please remember that, as discussed in the main text, the surface layer itself has another $\mathrm{Sm} 4 f$ valence and is insulating. The topological surface states hence appear in the subsurface layer in the unperturbed systems.

Figure 8 (left) clearly shows that one of the flat $4 f$ orbitals (i.e., the $j_{z}= \pm 5 / 2$ ) is shifted above the Fermi energy upon increasing the $j_{z}= \pm 5 / 2$ potential $V_{5 / 2}$. At the same time, the topological surface states around the $\Gamma$ point shift from the subsurface layer for $V_{5 / 2}=0$ to the sub-subsurface layer at $V_{5 / 2}=0.73 \mathrm{eV}$. There is a crossover in between with the surface states being extended to both layers.

At the same time the topological surface states around the $X$ point remain on the subsurface layer. If we instead apply a 


\section{Sm 4 f sub-surface}
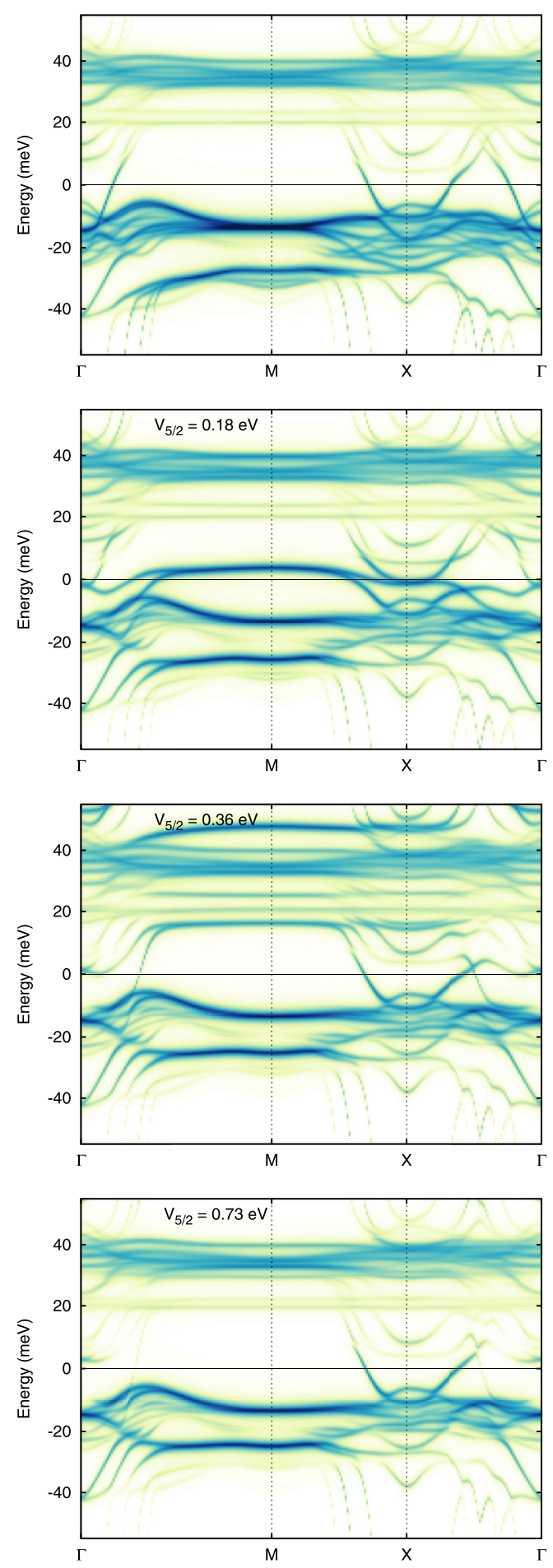

\section{Sm 4 f subsub-surface}
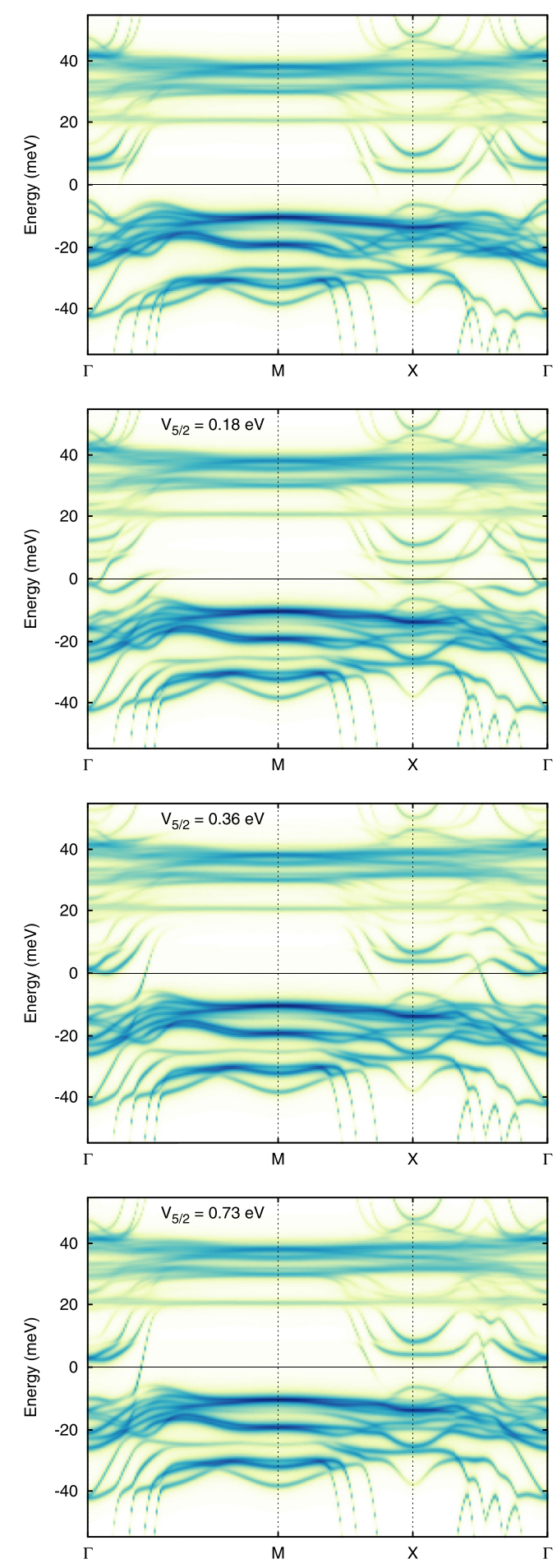

FIG. 8. The k-resolved DFT+DMFT spectrum in the presence of a perturbing potential at the subsurface $\operatorname{Sm~} 4 f J=5 / 2, j_{z}= \pm 5 / 2$ states of the Sm-terminated $\mathrm{SmB}_{6}$ supercell depicted in Fig. 10. The left (right) panel shows the projection upon the subsurface (sub-subsurface) Sm $4 f$ states. The topological surface states around the $\Gamma$ point shift from the subsurface to the sub-subsurface as the potential is increased.

potential to the $j_{z}= \pm 1 / 2$ spin orbitals as in Fig. 9, it is these topological surface states which start to shift to the next layer below. The $X$ pocket is however more robustly anchored to the subsurface atom compared to the $\Gamma$ pocket, and does not shift completely away even for $V=0.73 \mathrm{eV}$.
The larger "mobility" of the $\Gamma$ pocket in the $j_{z}=$ $\pm 5 / 2, \quad J=5 / 2$ spin orbitals is interesting, as it is seen in some experiments $[18,20,21]$ but not in others $[11,18,20]$. In the latter experiments, the $\Gamma$ pocket might simply hide a few layers deeper in the bulk, 
Sm $4 f$ sub-surface
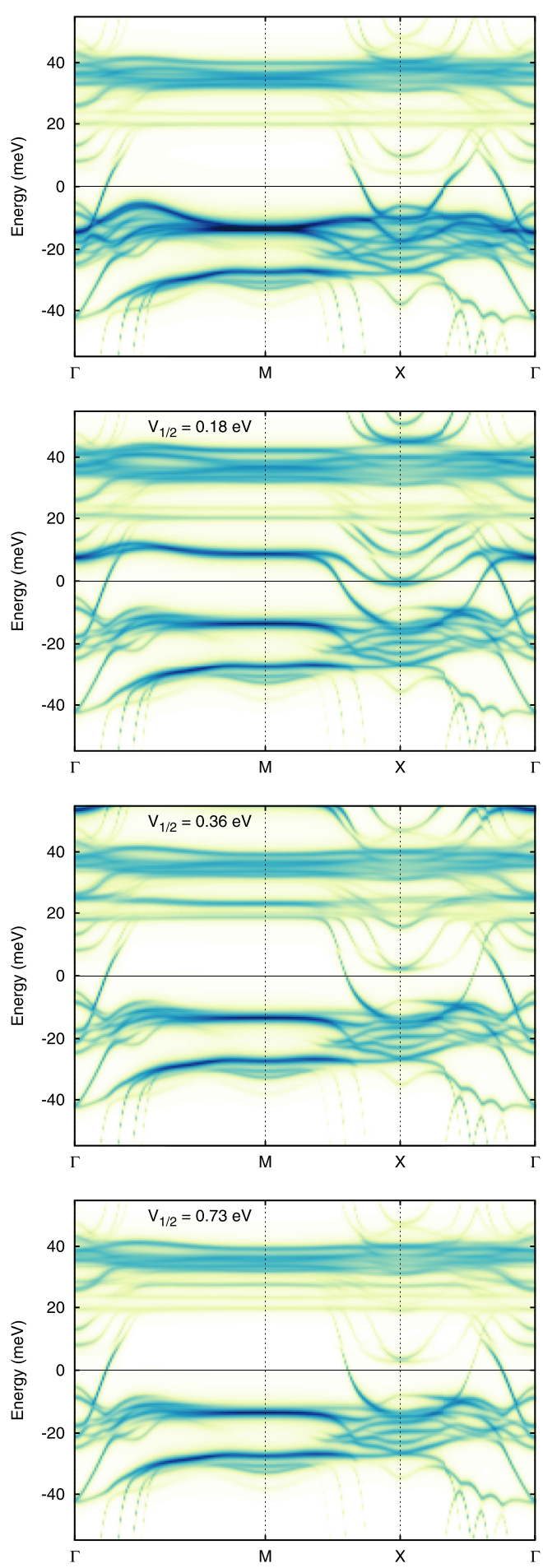

Sm $4 f$ subsub-surface
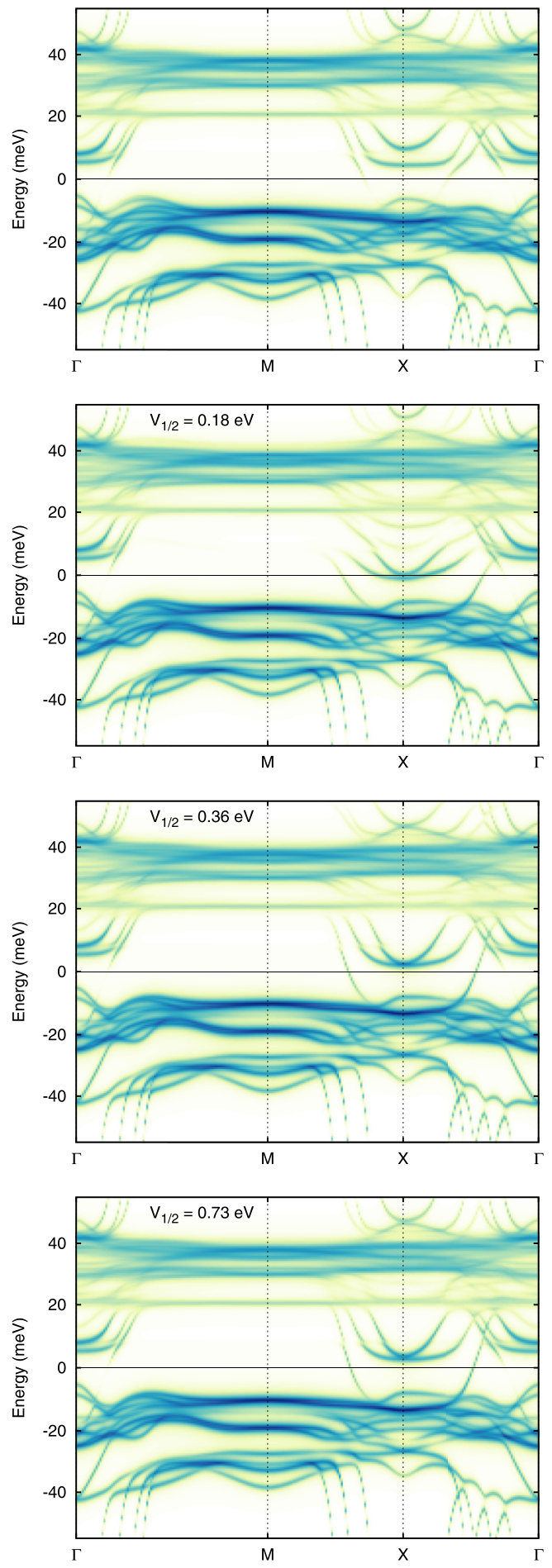

FIG. 9. Same as Fig. 8, but now the perturbing potential is applied to the subsurface $\operatorname{Sm~} 4 f J=5 / 2, j_{z}= \pm 1 / 2$ spin orbitals. The topological surface states around the $X$ point shift partially from the subsurface to the sub-subsurface as the potential is increased.

escaping its detection in surface-sensitive photoemission experiments.

Another possible explanation of the experimental discrepancies reveals itself at $V_{5 / 2}=0.36 \mathrm{eV}$ in Fig. 8. At this potential strength the topologically derived $X$ pocket and $\Gamma$ pocket get very close to each other around the Fermi energy, and the latter pocket can easily be misidentified as an umklapp state $[20,21]$. Interestingly, at the very same potential there are trivial bands which grace the Fermi energy close to the $\Gamma$ point. These trivial bands may be connected to the weak $\alpha$ pocket seen in some experiments [18,20,21]. However, to put these observations on firmer ground several fully 

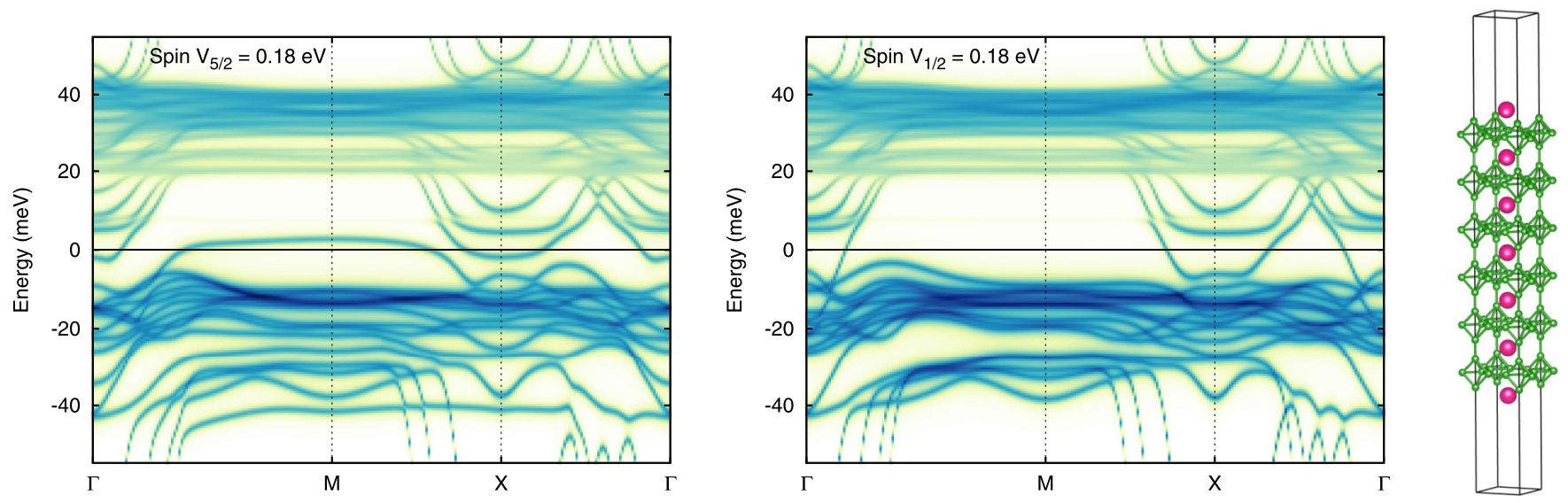

FIG. 10. The k-resolved DFT+DMFT spectrum (including all layers) of the Sm-terminated $\mathrm{SmB}_{6}$ supercell, shown in the right panel, in the presence of a spin-polarized $\left(B_{z}\right)$ time-reversal symmetry breaking field. The left (middle) panel shows when the potential is applied to the subsurface $\operatorname{Sm} 4 f J=5 / 2, j_{z}= \pm 5 / 2\left(j_{z}= \pm 1 / 2\right)$ spin orbitals. The left panel shows a clear separation between the conduction and the valence bands: the perturbing field destroys the topological protection of the surface bands. In the middle panel, the field is applied to the $j_{z}= \pm 1 / 2$ spin orbitals, and a smaller gap emerges around the $X$ point, while the $\Gamma$ pocket remains ungapped.

self-consistent slab calculations with various realistic surface reconstructions are needed, which is beyond the scope of current study.

\section{Magnetic field perpendicular to the surface}

Next we apply a spin-polarizing field instead of a potential term to the surface. The field $\left(B_{z}\right)$ is directed perpendicular to the surface and it breaks the time-reversal symmetry of the system. The perturbation is hence expected to destroy the topological protection of the surface states. We target the same two different pairs of states as for the potential term. That is, we apply the field to the subsurface $\operatorname{Sm} 4 f J=5 / 2$, $j_{z}= \pm 5 / 2$, and $j_{z}= \pm 1 / 2$ spin orbitals. As shown in Fig. 10, the perturbing fields indeed allow the surface states around the Fermi level to hybridize, including the otherwise protected topological surface states.
Let us start with the field applied to the $j_{z}= \pm 5 / 2$ spin orbitals in Fig. 10 (left). The large coupling between the field and the $j_{z}= \pm 5 / 2$ component of the surface states makes the surface bands very susceptible to the perturbing (time-reversal symmetry breaking) field. The topological surface states start to hybridize and an indirect band gap opens. Quantitatively, the spectrum is altered more at the $\Gamma$ point than at the $X$ point as here the $j_{z}= \pm 5 / 2$ character dominates. But also at the $X$ point a small gap opens.

It is exactly vice versa if we apply the perturbing field to the subsurface $\operatorname{Sm} 4 f J=5 / 2, j_{z}= \pm 1 / 2$ spin orbitals; see Fig. 10 (right). Here, actually only the topological surface state around the $X$ point is gapped out, whereas the topological surface bands around $\Gamma$ remain intact. While applying a field to only part of the $\operatorname{Sm} 4 f$ states is, as a matter of course, a theoretical construct, it still demonstrates that the $\Gamma$ and $X$ pockets can clearly shift independently.
[1] M. Z. Hasan and C. L. Kane, Rev. Mod. Phys. 82, 3045 (2010).

[2] X.-L. Qi and S.-C. Zhang, Rev. Mod. Phys. 83, 1057 (2011).

[3] B. A. Bernevig, T. L. Hughes, and S.-C. Zhang, Science 314, 1757 (2006).

[4] H. Zhang, C.-X. Liu, X.-L. Qi, X. Dai, Z. Fang, and S.-C. Zhang, Nat. Phys. 5, 438 (2009).

[5] Y. Xia, D. Qian, D. Hsieh, L. Wray, A. Pal, H. Lin, A. Bansil, D. Grauer, Y. S. Hor, R. J. Cava, and M. Z. Hasan, Nat. Phys. 5, 398 (2009).

[6] B. Bradlyn, L. Elcoro, J. Cano, M. G. Vergniory, Z. Wang, C. Felser, M. I. Aroyo, and B. A. Bernevig, Nature (London) 547, 298 (2017).

[7] R. O. Jones and O. Gunnarsson, Rev. Mod. Phys. 61, 689 (1989).

[8] J. P. Perdew and Y. Wang, Phys. Rev. B 45, 13244 (1992).

[9] M. Dzero, K. Sun, V. Galitski, and P. Coleman, Phys. Rev. Lett. 104, 106408 (2010).

[10] L. Fu and C. L. Kane, Phys. Rev. B 76, 045302 (2007).
[11] J. D. Denlinger, J. W. Allen, J.-S. Kang, K. Sun, B.-I. Min, D.-J. Kim, and Z. Fisk, JPS Conf. Proc. 3, 017038 (2014).

[12] Z. Wang and S.-C. Zhang, Phys. Rev. X 2, 031008 (2012).

[13] Y.-Y. He, H.-Q. Wu, Z. Y. Meng, and Z.-Y. Lu, Phys. Rev. B 93, 195164 (2016).

[14] S. Y. Savrasov, K. Haule, and G. Kotliar, Phys. Rev. Lett. 96, 036404 (2006).

[15] L. Wang, H. Jiang, X. Dai, and X. C. Xie, Phys. Rev. B 85, 235135 (2012).

[16] S. Wolgast, C. Kurdak, K. Sun, J. W. Allen, D.-J. Kim, and Z. Fisk, Phys. Rev. B 88, 180405(R) (2013).

[17] D. J. Kim, S. Thomas, T. Grant, J. Botimer, Z. Fisk, and J. Xia, Sci. Rep. 3, 3150 (2013).

[18] J. Jiang, S. Li, T. Zhang, Z. Sun, F. Chen, Z. Ye, M. Xu, Q. Ge, S. Tan, X. Niu, M. Xia, B. Xie, Y. Li, X. Chen, H. Wen, and D. Feng, Nat. Commun. 4, 3010 (2013).

[19] M. Neupane, N. Alidoust, S.-Y. Xu, T. Kondo, Y. Ishida, D. J. Kim, C. Liu, I. Belopolski, Y. J. Jo, T.-R. Chang, H.-T. Jeng, 
T. Durakiewicz, L. Balicas, H. Lin, A. Bansil, S. Shin, Z. Fisk, and M. Z. Hasan, Nat. Commun. 4, 2991 (2013).

[20] N. Xu, X. Shi, P. K. Biswas, C. E. Matt, R. S. Dhaka, Y. Huang, N. C. Plumb, M. Radović, J. H. Dil, E. Pomjakushina, K. Conder, A. Amato, Z. Salman, D. M. Paul, J. Mesot, H. Ding, and M. Shi, Phys. Rev. B 88, 121102(R) (2013).

[21] P. Hlawenka, K. Siemensmeyer, E. Weschke, A. Varykhalov, J. Sánchez-Barriga, N. Y. Shitsevalova, A. V. Dukhnenko, V. B. Filipov, S. Gabáni, K. Flachbart, O. Rader, and E. D. L. Rienks, Nat. Commun. 9, 517 (2018).

[22] Y. Ohtsubo, Y. Yamashita, K. Hagiwara, S.-i. Ideta, K. Tanaka, R. Yukawa, K. Horiba, H. Kumigashira, K. Miyamoto, T. Okuda, W. Hirano, F. Iga, and S.-i. Kimura, Nat. Commun. 10, 2298 (2019).

[23] N. Xu, P. K. Biswa, J. H. Dil, R. S. Dhaka, G. Landolt, S. Muff, C. E. Matt, X. Shi, N. C. Plumb, M. Radović, E. Pomjakushina, K. Conder, A. Amato, S. V. Borisenko, R. Yu, H.-M. Weng, Z. Fang, X. Dai, J. Mesot, H. Ding et al., Nat. Commun. 5, 4566 (2014).

[24] Z.-H. Zhu, A. Nicolaou, G. Levy, N. P. Butch, P. Syers, X. F. Wang, J. Paglione, G. A. Sawatzky, I. S. Elfimov, and A. Damascelli, Phys. Rev. Lett. 111, 216402 (2013).

[25] B. S. Tan, Y.-T. Hsu, B. Zeng, M. C. Hatnean, N. Harrison, Z. Zhu, M. Hartstein, M. Kiourlappou, A. Srivastava, M. D. Johannes, T. P. Murphy, J.-H. Park, L. Balicas, G. G. Lonzarich, G. Balakrishnan, and S. E. Sebastian, Science 349, 287 (2015).

[26] G. Li, Z. Xiang, F. Yu, T. Asaba, B. Lawson, P. Cai, C. Tinsman, A. Berkley, S. Wolgast, Y. S. Eo, D.-J. Kim, C. Kurdak, J. W. Allen, K. Sun, X. H. Chen, Y. Y. Wang, Z. Fisk, and L. Li, Science 346, 1208 (2014).

[27] J. D. Denlinger, S. Jang, G. Li, L. Chen, B. J. Lawson, T. Asaba, C. Tinsman, F. Yu, K. Sun, J. W. Allen, C. Kurdak, D.-J. Kim, Z. Fisk, and L. Li, arXiv:1601.07408.

[28] E. Frantzeskakis, N. de Jong, B. Zwartsenberg, Y. K. Huang, Y. Pan, X. Zhang, J. X. Zhang, F. X. Zhang, L. H. Bao, O. Tegus, A. Varykhalov, A. de Visser, and M. S. Golden, Phys. Rev. X 3, 041024 (2013).

[29] N. Wakeham, P. F. S. Rosa, Y. Q. Wang, M. Kang, Z. Fisk, F. Ronning, and J. D. Thompson, Phys. Rev. B 94, 035127 (2016).

[30] N. J. Laurita, C. M. Morris, S. M. Koohpayeh, P. F. S. Rosa, W. A. Phelan, Z. Fisk, T. M. McQueen, and N. P. Armitage, Phys. Rev. B 94, 165154 (2016).

[31] W. T. Fuhrman, J. R. Chamorro, P. A. Alekseev, J.-M. Mignot, T. Keller, J. A. Rodriguez-Rivera, Y. Qiu, P. Nikolić, T. M. McQueen, and C. L. Broholm, Nat. Commun. 9, 1539 (2018).

[32] A. Georges, G. Kotliar, W. Krauth, and M. J. Rozenberg, Rev. Mod. Phys. 68, 13 (1996).

[33] G. Kotliar, S. Y. Savrasov, K. Haule, V. S. Oudovenko, O. Parcollet, and C. A. Marianetti, Rev. Mod. Phys. 78, 865 (2006).

[34] K. Held, Adv. Phys. 56, 829 (2007).
[35] J. D. Denlinger, J. W. Allen, J.-S. Kang, K. Sun, J.-W. Kim, J. H. Shim, B. I. Min, D.-J. Kim, and Z. Fisk, arXiv:1312.6637.

[36] J. Kim, K. Kim, C.-J. Kang, S. Kim, H. C. Choi, J.-S. Kang, J. D. Denlinger, and B. I. Min, Phys. Rev. B 90, 075131 (2014).

[37] R. Peters, T. Yoshida, H. Sakakibara, and N. Kawakami, Phys. Rev. B 93, 235159 (2016).

[38] P. Thunström, I. Di Marco, A. Grechnev, S. Lebègue, M. I. Katsnelson, A. Svane, and O. Eriksson, Phys. Rev. B 79, 165104 (2009).

[39] A. B. Shick, L. Havela, A. I. Lichtenstein, and M. I. Katsnelson, Sci. Rep. 5, 15429 (2015).

[40] F. Lu, J. Zhao, H. Weng, J. Z. Fang, and X. Dai, Phys. Rev. Lett. 110, 096401 (2013).

[41] J. M. Wills, O. Eriksson, M. Alouani, and D. L. Price, in Electronic Structure and Physical Properties of Solids: The Uses of the LMTO Method, Lecture Notes in Physics Vol. 535, edited by H. Dreyssé (Springer-Verlag, Berlin, 2000), pp. 148-167.

[42] A. Grechnev, I. Di Marco, M. I. Katsnelson, A. I. Lichtenstein, J. Wills, and O. Eriksson, Phys. Rev. B 76, 035107 (2007).

[43] P. Thunström, I. Di Marco, and O. Eriksson, Phys. Rev. Lett. 109, 186401 (2012).

[44] H. Yamaoka, P. Thunström, I. Jarrige, K. Shimada, N. Tsujii, M. Arita, H. Iwasawa, H. Hayashi, J. Jiang, T. Habuchi, D. Hirayama, H. Namatame, M. Taniguchi, U. Murao, S. Hosoya, A. Tamaki, and H. Kitazawa, Phys. Rev. B 85, 115120 (2012).

[45] M. Caffarel and W. Krauth, Phys. Rev. Lett. 72, 1545 (1994).

[46] A. Liebsch and H. Ishida, J. Phys.: Condens. Matter 24, 053201 (2011).

[47] O. Grånäs, I. D. Marco, P. Thunström, L. Nordström, O. Eriksson, T. Björkman, and J. Wills, Comput. Mater. Sci. 55, 295 (2012).

[48] I. V. Solovyev, P. H. Dederichs, and V. I. Anisimov, Phys. Rev. B 50, 16861 (1994).

[49] V. I. Anisimov, J. Zaanen, and O. K. Andersen, Phys. Rev. B 44, 943 (1991).

[50] M. Mizumaki, S. Tsutsui, and F. Iga, J. Phys.: Conf. Ser. 176, 012034 (2009).

[51] M. Sundermann, H. Yavaş, K. Chen, D. J. Kim, Z. Fisk, D. Kasinathan, M. W. Haverkort, P. Thalmeier, A. Severing, and L. H. Tjeng, Phys. Rev. Lett. 120, 016402 (2018).

[52] A. Bansil, H. Lin, and T. Das, Rev. Mod. Phys. 88, 021004 (2016).

[53] C. Gramsch and M. Potthoff, Phys. Rev. B 92, 235135 (2015).

[54] The reason is simple: the symmetries of the system enforce that only local orbitals that share the same irreducible representation may hybridize.

[55] They have the same odd parity when the inversion center is placed at the Sm atom.

[56] This is the case when excluding the semicore and core states. 